Harvey Rosen, Department of Mathematics, University of Alabama, Tuscaloosa, AL 35487, e-mail: hrosen@@gp.as.ua.edu

\title{
EVERY REAL FUNCTION IS THE SUM OF TWO EXTENDABLE CONNECTIVITY FUNCTIONS
}

\begin{abstract}
It is shown that an arbitrary fucntion $f: \mathbb{R} \rightarrow \mathbb{R}$ can be written as the sum of two extendable connectivity functions.
\end{abstract}

Let $K$ be any one of the following three classes of functions from $\mathbb{R}$ into $\mathbb{R}$ : Darboux functions, connectivity functions, or almost continuous functions. It is known that an arbitrary function $f: \mathbb{R} \rightarrow \mathbb{R}$ is the sum of two functions belonging to $K[8],[3],[10],[2]$ and [7]. We show that this result is also true for the class $K$ of extendable connectivity functions. This answers a question of Gibson in [4]. Consequently, just like for the other classes, summation does not preserve for extendable functions any topological properties.

For a Darboux function $g: \mathbb{R} \rightarrow \mathbb{R}, g(C)$ is connected whenever $C$ is connected. Let $X=\mathbb{R}$ or $\mathbb{R}^{2}$. A function $G: X \rightarrow \mathbb{R}$ is called connectivity if the graph of the restriction $G \mid C$ is connected for each connected subset $C$ of $X$. According to [6], [14], [13], when $X=\mathbb{R}^{2}$, this last concept is equivalent to the notion of peripheral continuity, which means that for each $x \in X$ and each open neighborhood $U$ of $x$ and $V$ of $G(x)$, there exists an open neighborhood $W$ of $x$ in $U$ such that $G(\operatorname{bd}(W)) \subset V$, where $\operatorname{bd}(W)$ denotes the set-theoretic boundary of $W$ in $X$. We say $g: \mathbb{R} \rightarrow \mathbb{R}$ is an extendable connectivity function if there exists a connectivity function $G: \mathbb{R}^{2} \rightarrow \mathbb{R}$ such that $G(x, 0)=g(x)$ for all $x \in \mathbb{R}$, and we say a set $A \subset \mathbb{R}$ is $g$-negligible if every function from $\mathbb{R}$ into $\mathbb{R}$ obtained by arbitrarily redefining $g$ on $A$ is still an extendable connectivity function. Every open neighborhood of the graph of an almost continuous function $g: \mathbb{R} \rightarrow \mathbb{R}$ contains the graph of some continuous function from $\mathbb{R}$

Mathematical Reviews subject classification: Primary: 26A15; Secondary: 54C08

Received by the editors June 16,1995 
into $\mathbb{R}$. Let $I=[0,1]$. There is an extendable connectivity function $g: I \rightarrow I$ whose graph is dense in $I^{2}$ [1], [5] and [12]. Natkaniec remarks in [9] that it is unknown whether there exists an extendable connectivity function $g: I \rightarrow \mathbb{R}$ which is dense in $I \times \mathbb{R}$. We give such an example and use it and some results of Natkaniec [9] to verify the title.

Example 1 There exists an extendable connectivity function $g: \mathbb{R} \rightarrow \mathbb{R}$ whose graph is dense in $\mathbb{R}^{2}$.

Proof. We outline how to show this. Let $Q=\left\{r_{1}, r_{2}, r_{3}, \ldots\right\}$ be the set of rational numbers in $\mathbb{R} \times\{0\}$, and let

$$
\left\{d_{1}, d_{2}, d_{3}, d_{4}, d_{5}, d_{6}, \ldots\right\}=\{1,-1,2,-2,3,-3, \ldots\} .
$$

In what follows, a "triangle" $t$ will consist of the points interior to the three sides along with the points on its open base $b$. First we want to define partially a function $G: \mathbb{R} \times[0, \infty) \rightarrow \mathbb{R}$ which is peripherally continuous. For $n=$ $1,2,3, \ldots$ we let $T_{n}$ denote a countable collection of triangles $t_{i}$ in $\mathbb{R} \times[0, \infty)$ whose bases $b_{i}$ form a locally finite countable collection $B_{n}$ of open intervals of $\mathbb{R} \times\{0\}$ with irrational endpoints. Furthermore, let $G$ be a function such that

(1) $\operatorname{diam}\left(t_{i}\right)<\frac{1}{n}$,

(2) $T_{n+1}$ is a refinement of $T_{n}$ and $B_{n+1}$ is a refinement of $B_{n}$,

(3) each element $r_{j}$ of $\left\{r_{1}, r_{2}, \ldots, r_{n}\right\}$ belongs to exactly two members $b_{j}^{\prime}$, $b_{j}^{\prime \prime}$ of $B_{n}$ which are bases of triangles $t_{j}^{\prime}, t_{j}^{\prime \prime}$ in $T_{n}$ with $\operatorname{cl}\left(t_{j}^{\prime}\right) \subset t_{j}^{\prime \prime}$,

(4) $T_{n}^{*}=T_{n} \backslash\left\{t_{j}^{\prime \prime}: 1 \leq j \leq n\right\}$ is a "sawtooth" countable collection of disjoint triangles, $B_{n}^{*}=B_{n} \backslash\left\{b_{j}^{\prime \prime}: 1 \leq j \leq n\right\}$ is a countable collection of disjoint open intervals, and $\mathbb{R} \times\{0\}=\cup\left\{\operatorname{cl}\left(b_{i}\right): b_{i} \in B_{n}^{*}\right\}$,

(5) for $1 \leq j \leq n, G\left(\operatorname{bd}\left(t_{j}^{\prime}\right) \backslash b_{j}^{\prime}\right)=d_{n}$ and $G\left(\operatorname{bd}\left(t_{j}^{\prime \prime}\right) \backslash b_{j}^{\prime \prime}\right)=0$,

(6) for each $t_{i} \in T_{n}$ the variation of $G(x)$ on $\operatorname{bd}\left(t_{i}\right) \backslash b_{i}$ is $<\frac{1}{n}$, and

(7) $G$ maps the closed set $(\mathbb{R} \times[0, \infty)) \backslash \cup T_{n}^{*}$ continuously onto $\left[-d_{n}+1, d_{n}\right]$ if $n$ is odd or onto $\left[-\left|d_{n}\right|,\left|d_{n}\right|\right]$ if $n$ is even.

Here is how to attain condition (6) for $n>1$. Let $E$ denote the set of endpoints of all intervals belonging to $B_{n-1}$ along with the endpoints of each $b_{j}^{\prime}, b_{j}^{\prime \prime} \in B_{n}$ described in (3) for each $r_{j} \in\left\{r_{1}, r_{2}, \ldots, r_{n}\right\}$. Suppose $c$ and $d$ are consecutive points of $E$ with $r_{j} \notin(c, d)$ for $1 \leq j \leq n$. Even if $|G(d)-G(c)|$ 
is a large value, a sufficient number of consecutive small triangles of diameter $<\frac{1}{n}$ which are to belong to $T_{n}$ can be constructed forming sawteeth from $c$ to $d$ so that the variation of $G(x)$ on the slanted sides of each of these triangles will be less than $\frac{1}{n}$. We may suppose $G(x)$ varies monotonically from $G(c)$ to $G(d)$ along the slanted edges of the sawteeth from $c$ to $d$.

We now define $G$ on the rest of $\mathbb{R} \times\{0\}$. Let $\varepsilon>0$.

Case (i): $x$ is a rational number $r_{j} \in \mathbb{R} \times\{0\}$. Then define $G(x)=0$. For each $n \geq j$, there exist by (2), (3), and (5), open intervals $b_{j}^{\prime}, b_{j}^{\prime \prime} \in B_{n}$ such that $G\left(\operatorname{bd}\left(t_{j}^{\prime}\right) \backslash b_{j}^{\prime}\right)=d_{n}$ and $G\left(\operatorname{bd}\left(t_{j}^{\prime \prime}\right) \backslash b_{j}^{\prime \prime}\right)=0$. So $\operatorname{diam}\left(\{G(x)\} \cup G\left(\operatorname{bd}\left(t_{j}^{\prime \prime}\right) \backslash b_{j}^{\prime \prime}\right)=\right.$ $\operatorname{diam}(\{0\})=0<\varepsilon$.

Case (ii): $x$ is an irrational number in $\mathbb{R} \times\{0\}$ that is not an endpoint of any $b_{i}$ in any $B_{n}$. Suppose there exists an integer $N$ such that for all $n>N, x$ does not belong to any $b_{j}^{\prime \prime} \in B_{n}, 1 \leq j \leq n$. Then by (3), (5) and (6), for each $n>N$, there exists in $T_{n}$ a triangle $t_{i}$ whose base $b_{i}$ contains $x$ and on whose slanted sides the value of $G$ lies in $\left[-\left|d_{N}\right|,\left|d_{N}\right|\right]$. For each $n>N$, choose a point $x_{n}$ belonging to a slanted side of $t_{i}$. Then there exists a cluster point $y$ of the sequence $G\left(x_{1}\right), G\left(x_{2}\right), G\left(x_{3}\right), \ldots$, and so define $G(x)=y$. By (1) and (6), $\operatorname{diam}\left(\{G(x)\} \cup G\left(\operatorname{bd}\left(t_{i}\right) \backslash b_{i}\right)\right)<\epsilon$ for infinitely many $n$. On the other hand, if we suppose there does not exist such an integer $N$, then there are infinitely many $n$ such that $x \in b_{j}^{\prime \prime} \in B_{n}$ for some $j$ with $1 \leq j \leq n$. Because of $(5), G\left(\operatorname{bd}\left(t_{j}^{\prime \prime}\right) \backslash b_{j}^{\prime \prime}\right)=0$, and so define $G(x)=0$. Therefore $\operatorname{diam}\left(\{G(x)\} \cup\left(G\left(\operatorname{bd}\left(t_{j}^{\prime \prime}\right) \backslash b_{j}^{\prime \prime}\right)\right)=0<\epsilon\right.$.

Case (iii): $x$ is an irrational number in $\mathbb{R} \times\{0\}$ that is an endpoint of some interval belonging to some $B_{m}$. Then $G(x)$ is already defined. By (2) and (4), for each $n \geq m, x$ is an endpoint of adjacent intervals $b_{i}$ and $b_{k}$ in $B_{n}$. Because of (1) and (6), there exists an $n \geq m$ such that $\frac{2}{n}<\epsilon, t_{i} \cup t_{k}$ has diameter $<\frac{2}{n}$, and the variation of $G$ on $\operatorname{bd}\left(t_{i} \cup t_{k}\right) \backslash\left(b_{i} \cup b_{k}\right)$ is $<\frac{2}{n}$. Since, by (7), $G$ restricted to $(\mathbb{R} \times[0, \infty)) \backslash \cup T_{n}^{*}$ is continuous at $x$, there exists an open disk $D$ centered at $x$ and not containing the other vertices of $t_{i}$ and $t_{k}$ such that the diameter of the open neighborhood $U=t_{i} \cup t_{k} \cup(D \cap(\mathbb{R} \times[0, \infty)))$ of $x$ in $\mathbb{R} \times[0, \infty)$ is $<\frac{2}{n}$ and $\operatorname{diam}(\{G(x)\} \cup G(\operatorname{bd}(U)))<\frac{2}{n}<\epsilon$.

Case (iv): $x \in \mathbb{R} \times(0, \infty)$. According to (7), $G$ is already defined and continuous at $x$.

For each case, we have $G$ is peripherally continuous at $x$. We can extend $G$ to a peripherally continuous function $G: \mathbb{R}^{2} \rightarrow \mathbb{R}$ by defining $G(s, t)=$ $G(s,-t)$ whenever $(s, t) \in \mathbb{R} \times(-\infty, 0)$. On account of (5), the extendable connectivity function $g: \mathbb{R} \rightarrow \mathbb{R}$ defined by $g(x)=G(x, 0)$ for $x \in \mathbb{R}$ has its graph dense in $\mathbb{R}^{2}$.

Theorem 1 Let $f: \mathbb{R} \rightarrow \mathbb{R}$ be an arbitrary function. Then $f=g_{1}+g_{2}$ for functions $g_{1}, g_{2}: \mathbb{R} \rightarrow \mathbb{R}$ which are extendable connectivity functions. 
Proof. First let $g: \mathbb{R} \rightarrow \mathbb{R}$ be the above example of an extendable connectivity function whose graph is dense in $\mathbb{R} \times \mathbb{R}$. It follows from Theorem 1 in [11] that there exists a dense $G_{\delta}$ subset $A$ of $\mathbb{R}$ that is $g$-negligible. Since $\mathbb{R} \backslash A$ is of the first category, it follows from Lemma 3 in [9] that there exists a homeomorphism $h: \mathbb{R} \rightarrow \mathbb{R}$ such that $(\mathbb{R} \backslash A \cap h(\mathbb{R} \backslash A)=\emptyset$. Therefore $h(\mathbb{R} \backslash A) \subset A$; i.e., $\mathbb{R} \backslash A \subset h^{-1}(A)$. According to Corollary 1 and Lemma 2 (which still hold when $\mathbb{R}$ replaces $I$ and $J$ there) in [9], $g \circ h$ is an extendable connectivity function and $h^{-1}(A)$ is $g \circ h$-negligible. So $\mathbb{R} \backslash A$ is $g \circ h$-negligible. Define extendable connectivity functions $g_{1}, g_{2}: \mathbb{R} \rightarrow \mathbb{R}$ by

$$
g_{1}=\left\{\begin{array}{ll}
g \circ h & \text { on } A \\
f-g & \text { on } \mathbb{R} \backslash A
\end{array} \quad \text { and } \quad g_{2}= \begin{cases}f-(g \circ h) & \text { on } A \\
g & \text { on } \mathbb{R} \backslash A .\end{cases}\right.
$$

Then $f=g_{1}+g_{2}$.

Question 1 If $f: I \rightarrow I$ is an arbitrary bounded function, does $f=g_{1}+g_{2}$, where $g_{1}$ and $g_{2}$ are bounded extendable connectivity functions? Natkaniec has shown that $f$ is the sum of three such functions $g_{1}, g_{2}, g_{3}$ [9].

Analogous results have been obtained by Ciesielski and Reclaw in their paper, Cardinal invariants concerning extendable and peripherally continuous functions, and according to the referee, a negative answer to the above question follows from latest results of Ciesielski and Maliszewski.

\section{References}

[1] J. B. Brown, Totally discontinuous connectivity functions, Colloq. Math. 23 (1971), 53-60.

[2] A. M. Bruckner and J. G. Ceder, On jumping functions by connected sets, Czech. Math. J. 22 (1972), 435-448.

[3] H. Fast, Une remarque sur la propriete de Weierstrass, Colloq. Math. 7 (1959), 75-77.

[4] R. G. Gibson, A property of Borel measurable functions and extendable functions, Real Analysis Exch. 13 (1987-88), 11-15.

[5] R. G. Gibson, and F. Roush, Connectivity functions defined on $I^{n}$, Colloq. Math. 55 (1988), 41-44.

[6] M. R. Hagan, Equivalence of connectivity maps and peripherally continuous transformations, Proc. Amer. Math. Soc. 17 (1966), 175-177. 
[7] K. R. Kellum, Sums and limits of almost continuous functions, Colloq. Math. 31 (1974), 125-128.

[8] A. Lindenbaum, Sur quelques proprietes des fonctions de variable reelle, Ann. Soc. Polon. Math. 6 (1927), 129.

[9] T. Natkaniec, Extendability and almost continuity, preprint.

[10] D. Phillips, Real functions having graphs connected and dense in the plane, Fund. Math. 75 (1972), 47-49.

[11] H. Rosen, Limits and sums of extendable connectivity functions, Real Analysis Exch. 20 (1994-95), 183-191.

[12] H. Rosen, R. G. Gibson, and F. Roush, Extendable functions and almost continuous functions with a perfect road, Real Analysis Exch. 17 (199192), 248-257.

[13] J. R. Stallings, Fixed point theorems for connectivity maps, Fund. Math. 47 (1959), 249-263.

[14] G. T. Whyburn, Connectivity of peripherally continuous functions, Proc. Nat. Acad. Sci. U.S.A. 55 (1966), 1040-1041. 